\title{
Some relations involving a generalized fractional derivative operator
}

\author{
Sebastien Gaboury ${ }^{1 *}$, Richard Tremblay ${ }^{1}$ and Benoît-Jean Fugère ${ }^{2}$
}

"Correspondence:

s1gabour@uqac.ca

${ }^{1}$ Department of Mathematics and

Computer Science, University of

Quebec at Chicoutimi, Quebec,

G7H 2B1, Canada

Full list of author information is

available at the end of the article

\begin{abstract}
Recently, Katugampola (Appl. Math. Comput. 218:860-865, 2011) studied a special case of the Erdélyi-Kober generalized fractional derivative. This special case generalized the well-known Riemann-Liouville and the Hadamard fractional integrals into a single form. Katugampola denoted this special case by the operator ${ }_{0}^{\rho} D_{x}^{\alpha}$. Some properties and examples for this fractional derivative operator was given. In this paper, we present some additional properties for this operator defined, this time, in the complex plane. In particular, we express this fractional derivative operator in terms of the classical Riemann-Liouville fractional derivative operator. A generalized Leibniz rule is obtained.
\end{abstract}

MSC: $26 \mathrm{~A} 33 ; 33 \mathrm{C} 45$

Keywords: fractional derivatives; Pochhammer contour; Leibniz rules; special functions; summation formula

\section{Introduction}

One of the most frequently encountered operators of fractional derivatives, that is, calculus of integrals and derivatives of an arbitrary real or complex order is provided by the Riemann-Liouville operator $[1,2]$ denoted $D_{z}^{\alpha}$ and defined by

$$
D_{z}^{\alpha} z^{p} f(z):= \begin{cases}\frac{1}{\Gamma(-\alpha)} \int_{0}^{z} f(\zeta) \zeta^{p}(z-\zeta)^{-\alpha-1} \mathrm{~d} \zeta & \text { if } \operatorname{Re}(\alpha)<0, \\ \frac{d^{m}}{d z^{m}} D_{z}^{\alpha-m} z^{p} f(z) & \text { if } m-1 \leq \operatorname{Re}(\alpha)<m\end{cases}
$$

$(m \in \mathbb{N})$ which is valid for $\operatorname{Re}(p)>-1, z \neq 0$ on a simply connected region $\mathcal{R}$ containing the origin. Note that $f(z)$ must be analytic in $\mathcal{R}$ and specially at the origin.

Many other representations for fractional derivative operator exists. For example, using a single-loop contour of integration, we can obtain a less restrictive definition for the fractional derivative operator $D_{z}^{\alpha}$ which holds for $\operatorname{Re}(p)>-1$ and $\alpha$ not a negative integer. This representation has been widely used in many interesting papers [3-8]. The less restrictive representation for the fractional derivative operator in the complex plane is the one introduced by Lavoie, Osler and Tremblay [9] in 1976 based on Pochhammer's contour of integration [10-12]. This representation is valid when $\alpha$ is not a negative integer and $p$ is not an integer.

Many interesting applications of Pochhammer's contour based definition for fractional derivatives can be found in [13-17].

c) 2013 Gaboury et al.; licensee Springer. This is an Open Access article distributed under the terms of the Creative Commons Attribution License (http://creativecommons.org/licenses/by/2.0), which permits unrestricted use, distribution, and reproduction in any medium, provided the original work is properly cited. 
Another interesting and largely studied fractional derivative operator is the ErdélyiKober operator $[2,18]$ defined as follows:

$$
D_{0+; \rho ; \eta}^{\alpha} f(x):= \begin{cases}\frac{\rho x^{-\rho(-\alpha+\eta)}}{\Gamma(-\alpha)} \int_{0}^{x} \frac{f(t) t^{\rho \eta+\rho-1}}{\left(x^{\rho}-t^{\rho} \alpha^{\alpha+1}\right.} \mathrm{d} t & \text { if } \operatorname{Re}(\alpha)<0, \\ x^{-\rho \eta}\left(\frac{1}{\rho x^{\rho-1}} \frac{d}{d x}\right)^{m} x^{\rho(m+\eta)} D_{0+; \rho ; \eta-\alpha}^{\alpha-m} f(x) & \text { if } m-1 \leq \operatorname{Re}(\alpha)<m,\end{cases}
$$

where $\eta \in \mathcal{R}$ and $\rho>0$. This operator has been introduced to solve some integral equations of particular type. For many interesting applications of fractional calculus operators, the reader is referred to the book of Kilbas et al. [19].

Recently, Katugampola in [20] studied a special case of the Erdélyi-Kober generalized fractional derivative operator which contains as special cases the Riemann-Liouville fractional operator (1) and the Hadamard fractional operator [21]. In particular, the author considered the fractional derivative operator ${ }_{0}^{\rho} D_{x}^{\alpha}$ defined by

$$
{ }_{0}^{\rho} D_{x}^{\alpha} f(x):= \begin{cases}\frac{(\rho+1)^{\alpha+1}}{\Gamma(-\alpha)} \int_{0}^{x} \frac{f(t) t^{\rho}}{\left(x^{\rho+1}-t^{\rho+1}\right)^{\alpha+1}} \mathrm{~d} t & \text { if } \operatorname{Re}(\alpha)<0, \\ \left(x^{-\rho} \frac{d}{d x}\right)^{m \rho}{ }_{0}^{\alpha} D_{x}^{\alpha-m} f(x) & \text { if } m-1 \leq \operatorname{Re}(\alpha)<m\end{cases}
$$

$(m \in \mathbb{N})$ which is valid for $\rho \in \mathbb{R}$ with $\rho>-1$.

It is easy to verify that they are related by the following relation:

$$
{ }_{0}^{\rho} D_{x}^{\alpha} f(x)=(\rho+1)^{\alpha} x^{-(\rho+1) \alpha} D_{0+; \rho+1 ; 0}^{\alpha} f(x) .
$$

Now, substituting $\rho=0$ in (3) gives the standard Riemann-Liouville derivative (1). Moreover, considering functions belonging to a particular space of function, namely $X_{c}^{p}(a, b)$ $(c \in \mathbb{R}, 1 \leq p \leq \infty)$ (see, [21]) and taking the limit when $\rho \rightarrow-1^{+}$in (3), the author in [20] obtained, with the help of the L'Hospital's rule, the following relationship:

$$
\begin{aligned}
& \lim _{\rho \rightarrow-1^{+}} \frac{(\rho+1)^{\alpha+1}}{\Gamma(-\alpha)} \int_{0}^{x} \frac{f(t) t^{\rho}}{\left(x^{\rho+1}-t^{\rho+1}\right)^{\alpha+1}} \mathrm{~d} t \\
& =\frac{1}{\Gamma(-\alpha)} \int_{0}^{x} \lim _{\rho \rightarrow-1^{+}} f(t) t^{\rho}\left(\frac{x^{\rho+1}-t^{\rho+1}}{\rho+1}\right)^{-\alpha-1} \mathrm{~d} t \\
& =\frac{1}{\Gamma(-\alpha)} \int_{0}^{x}\left(\log \frac{x}{t}\right)^{-\alpha-1} \frac{f(t)}{t} \mathrm{~d} t .
\end{aligned}
$$

This last relation is the well-known and extensively studied Hadamard fractional integral [21-23].

Applying the generalized fractional derivative operator defined in (3) to the function $f(x)=x^{\nu}, v \in \mathbb{R}$ in the case $\operatorname{Re}(\alpha)<0$ yields the following formula:

$$
\begin{aligned}
{ }_{0}^{\rho} D_{x}^{\alpha} x^{\nu} & =\frac{(\rho+1)^{\alpha+1}}{\Gamma(-\alpha)} \int_{0}^{x} t^{\rho+\nu}\left(x^{\rho+1}-t^{\rho+1}\right)^{-\alpha-1} \mathrm{~d} t \\
& =\frac{(\rho+1)^{\alpha+1}}{\Gamma(-\alpha)} \int_{0}^{1} \frac{x^{\nu-\alpha(\rho+1)} u^{\frac{\nu}{\rho+1}}(1-u)^{-\alpha-1}}{\rho+1} \mathrm{~d} u \\
& =\frac{(\rho+1)^{\alpha} x^{\nu-\alpha(\rho+1)}}{\Gamma(-\alpha)} B\left(\frac{v}{\rho+1}+1,-\alpha\right)=\frac{(\rho+1)^{\alpha} \Gamma\left(\frac{\nu}{\rho+1}+1\right)}{\Gamma\left(\frac{v}{\rho+1}+1-\alpha\right)} x^{\nu-\alpha(\rho+1) .}
\end{aligned}
$$


The last relation is valid for $\frac{v}{\rho+1}>-1$. Note that we used the following change of variables $u=\frac{t^{\rho+1}}{x^{\rho+1}}$ in $(6)$ and that $B(x, y)$ holds for the familiar Beta function [2, p.17, Eq. 1.68]. Replacing $\rho$ by 0 in (6) gives the classical fractional integral formula for the function $x^{\nu}$, that is,

$$
D_{x}^{\alpha} x^{\nu}=\frac{\Gamma(1+v)}{\Gamma(1+v-\alpha)} x^{\nu-\alpha}
$$

with $\operatorname{Re}(\alpha)<0$ and $v>-1$. In this paper, we consider the fractional derivative operator ${ }_{0}^{\rho} D_{z}^{\alpha}$ defined in the complex plane. In Section 2, we obtain two representations for this fractional derivative operator in terms of the classical Riemann-Liouville fractional operator. The second representation is obtained by making use of a new transformation formula for the fractional derivative recently published by Tremblay et al. [16]. Some special cases are computed. Finally, in Section 3, we derive a generalized Leibniz rule for the fractional derivative operator ${ }_{0}^{\rho} D_{z}^{\alpha}$. Some applications of this Leibniz rule are also obtained.

\section{Representations of the generalized fractional derivative operator in terms of the classical Riemann-Liouville fractional derivative operator}

The purpose of this section is to develop two representations of the generalized fractional derivative operator ${ }_{0}^{\rho} D_{z}^{\alpha}$ in terms of the common Riemann-Liouville fractional operator (1). These representations are found by making use of a new transformation formula for fractional derivatives published recently by the authors [16]. Some examples of possible new relationships are also given.

Note that for the remainder of this paper, $(\lambda)_{n}$ will denote the Pochhammer's symbol defined by

$$
(\lambda)_{n}:=\frac{\Gamma(\lambda+n)}{\Gamma(\lambda)} ; \quad(\lambda)_{0}=1 .
$$

Moreover, we adopt the following notation to denote the generalized hypergeometric function

$$
{ }_{p} F_{q}\left[\begin{array}{cccc}
a_{1}, & a_{2}, & \ldots, & a_{p} ; \\
b_{1}, & b_{2}, & \ldots, & b_{q} ;
\end{array}\right]=\sum_{k=0}^{\infty} \frac{\left(a_{1}\right)_{k}\left(a_{2}\right)_{k} \cdots\left(a_{p}\right)_{k} z^{k}}{\left(b_{1}\right)_{k}\left(b_{2}\right)_{k} \cdots\left(b_{q}\right)_{k} k !} .
$$

The first representation for the fractional derivative operator ${ }_{0}^{\rho} D_{z}^{\alpha}$ is contained in the next theorem.

\section{Theorem 1}

(i) Let $\mathcal{R}$ be a simply connected region containing the origin.

(ii) Let $f(z)$ be analytic in $\mathcal{R}$. Then, for $\alpha$ and $\rho \in \mathbb{C}$ with $\operatorname{Re}(\rho)>-1$, the following relation holds true

$$
\begin{aligned}
{ }_{0}^{\rho} D_{z}^{\alpha} f(z)= & \frac{(\rho+1)^{\alpha+1}}{\Gamma(-\alpha)} z^{-(\rho+1)(\alpha+1)} \sum_{n=0}^{\infty} \frac{(\alpha+1)_{n}}{n !} z^{-n(\rho+1)} \\
& \times\left.\Gamma((\rho+1) n+\rho+1) D_{z}^{-(\rho+1) n-\rho-1} f(w-z)\right|_{w=z} .
\end{aligned}
$$


Proof Consider the integral representation of the operator ${ }_{0}^{\rho} D_{z}^{\alpha}$ in the complex plane with $\operatorname{Re}(\alpha)<0$ :

$$
{ }_{0}^{\rho} D_{z}^{\alpha} f(z)=\frac{(\rho+1)^{\alpha+1}}{\Gamma(-\alpha)} \int_{0}^{z} \frac{f(t) t^{\rho}}{\left(z^{\rho+1}-t^{\rho+1}\right)^{\alpha+1}} \mathrm{~d} t .
$$

Making the following change of variables $t=z-\xi(\mathrm{d} t=-\mathrm{d} \xi)$, we have

$$
\begin{aligned}
{ }_{0}^{\rho} D_{z}^{\alpha} f(z)= & -\frac{(\rho+1)^{\alpha+1}}{\Gamma(-\alpha)} \int_{z}^{0} \frac{f(z-\xi)(z-\xi)^{\rho}}{\left(z^{\rho+1}-(z-\xi)^{\rho+1}\right)^{\alpha+1}} \mathrm{~d} \xi \\
= & \frac{(\rho+1)^{\alpha+1}}{\Gamma(-\alpha)} z^{-(\rho+1)(\alpha+1)} \int_{0}^{z} \frac{f(z-\xi)(z-\xi)^{\rho}}{\left(1-\left(\frac{z-\xi}{z}\right)^{\rho+1}\right)^{\alpha+1}} \mathrm{~d} \xi \\
= & \frac{(\rho+1)^{\alpha+1} \Gamma(1+\rho)}{\Gamma(-\alpha)} z^{-(\rho+1)(\alpha+1)} \\
& \times\left. D_{z}^{-\rho-1}\left(1-\left(\frac{w-z}{w}\right)^{\rho+1}\right)^{-\alpha-1} f(w-z)\right|_{w=z} .
\end{aligned}
$$

Note that we must have $w \rightarrow z$ in the right side of (12) after the evaluation of the fractional derivative. So, the point $w$ must be near the point $z$. Using this fact, we can expand the expression $\left(1-\left(\frac{w-z}{w}\right)^{\rho+1}\right)^{-\alpha-1}$ in power series. We thus have

$$
\begin{aligned}
{ }_{0}^{\rho} D_{z}^{\alpha} f(z) & =\frac{(\rho+1)^{\alpha+1}}{\Gamma(-\alpha)} z^{-(\rho+1)(\alpha+1)} \int_{0}^{z} \sum_{n=0}^{\infty} \frac{(\alpha+1)_{n}}{n !}\left(\frac{z-\xi}{z}\right)^{(\rho+1) n} f(z-\xi)(z-\xi)^{\rho} \mathrm{d} \xi \\
& =\frac{(\rho+1)^{\alpha+1}}{\Gamma(-\alpha)} z^{-(\rho+1)(\alpha+1)} \sum_{n=0}^{\infty} \frac{(\alpha+1)_{n}}{n !} z^{-n(\rho+1)} \int_{0}^{z} f(z-\xi)(z-\xi)^{(\rho+1) n+\rho} \mathrm{d} \xi .
\end{aligned}
$$

Rewriting the integral in (13) in terms of the Riemann-Liouville fractional derivative operator yields the desired result.

Corollary 2 Substituting $\rho=0$ in Theorem 1 , we obtain

$$
D_{z}^{\alpha} f(z)=\left.\frac{z^{-(\alpha+1)}}{\Gamma(-\alpha)} \sum_{n=0}^{\infty}(\alpha+1)_{n} z^{-n} D_{z}^{-n-1} f(w-z)\right|_{w=z} .
$$

Example 1 Let $f(z)=(1-z)^{-\gamma}$ in Theorem 1. We obtain for the l.h.s. of (10) by making use of the power series expansion of $(1-z)^{-\gamma}$

$$
{ }_{0}^{\rho} D_{z}^{\alpha}(1-z)^{-\gamma}=\sum_{k=0}^{\infty} \frac{(\gamma)_{k} \rho}{k !} D_{z}^{\alpha} z^{k}=(\rho+1)^{\alpha} z^{-\alpha(\rho+1)} \sum_{k=0}^{\infty} \frac{(\gamma)_{k}}{k !} \frac{\Gamma\left(\frac{k}{\rho+1}+1\right)}{\Gamma\left(\frac{k}{\rho+1}+1-\alpha\right)} z^{k} .
$$

For the fractional derivative operator involved in the r.h.s. of (10), we have

$$
\begin{aligned}
& \left.D_{z}^{-(\rho+1) n-\rho-1}(1-(w-z))^{-\gamma}\right|_{w=z} \\
& \quad=\left.D_{z}^{-(\rho+1) n-\rho-1}(1-w)^{-\gamma}\left(1-\left(\frac{z}{w-1}\right)\right)^{-\gamma}\right|_{w=z}
\end{aligned}
$$




$$
\begin{aligned}
& =\left.(1-z)^{-\gamma} D_{z}^{-(\rho+1) n-\rho-1} \sum_{k=0}^{\infty} \frac{(\gamma)_{k}}{k !} \frac{z^{k}}{(w-1)^{k}}\right|_{w=z} \\
& =(1-z)^{-\gamma} \sum_{k=0}^{\infty} \frac{(\gamma)_{k}}{k !(z-1)^{k}} D_{z}^{-(\rho+1) n-\rho-1} z^{k} \\
& =(1-z)^{-\gamma} z^{(\rho+1) n+\rho+1} \sum_{k=0}^{\infty} \frac{(\gamma)_{k} \Gamma(1+k)}{\Gamma(2+k+(\rho+1) n+\rho) k !}\left(\frac{z}{z-1}\right)^{k} \\
& =\frac{(1-z)^{-\gamma} z^{(\rho+1) n+\rho+1}}{\Gamma(2+(\rho+1) n+\rho)}{ }^{k} F_{1}\left[\begin{array}{c}
\gamma, \quad \frac{z}{z-1} \\
2+(\rho+1) n+\rho ;
\end{array}\right] .
\end{aligned}
$$

Combining (15) and (16) in (10), we obtain after some simple calculations

$$
\begin{aligned}
& \sum_{k=0}^{\infty} \frac{(\gamma)_{k} \Gamma\left(\frac{k}{\rho+1}+1\right)}{\Gamma\left(\frac{k}{\rho+1}+1-\alpha\right)} \frac{z^{k}}{k !} \\
& \quad=\frac{(1-z)^{-\gamma}}{\Gamma(-\alpha)} \sum_{n=0}^{\infty} \frac{(\rho+1)(\alpha+1)_{n}}{n !((\rho+1) n+\rho+1)}{ }_{2} F_{1}\left[\begin{array}{ccc}
\gamma, & 1 ; \\
2+(\rho+1) n+\rho ;
\end{array}\right]
\end{aligned}
$$

where ${ }_{2} F_{1}\left[\begin{array}{c}a, b ; \\ c ;\end{array}\right]$ denotes the Gauss hypergeometric function [24].

Moreover, setting $\rho=0$ in (17) gives the following relationship involving the Gauss hypergeometric function:

$$
{ }_{2} F_{1}\left[\begin{array}{ccc}
\gamma, & 1 ; & \\
& 1-\alpha ;
\end{array}\right]=\frac{\Gamma(1-\alpha)(1-z)^{-\gamma}}{\Gamma(-\alpha)} \sum_{n=0}^{\infty} \frac{(\alpha+1)_{n}}{(n+1) !}{ }_{2} F_{1}\left[\begin{array}{cc}
\gamma, & 1 ; \\
& \\
2+n ; & \frac{z}{z-1}
\end{array}\right] .
$$

Recently, Tremblay et al. [16] discovered a new transformation formula for the fractional derivatives. Many interesting applications of this formula has also been given. Especially, they proved the next result.

Theorem 3 Let $f(z)$ be an analytic function in the simply connected region $\mathcal{R}$ containing the origin. For $\operatorname{Re}(\mu)>-1$, we have

$$
D_{z}^{\alpha} z^{\mu} f(z)=\left.\frac{\Gamma(1+\mu)}{\Gamma(-\alpha)} D_{z}^{-\mu-1}(z)^{-\alpha-1} f(w-z)\right|_{w=z} .
$$

Note that we must have $w \rightarrow z$ in the right side of (19) after the evaluation of the fractional derivative since the point $w$ must be near the point $z$.

With the help of this new result, we can easily obtain the next theorem.

\section{Theorem 4}

(i) Let $\mathcal{R}$ be a simply connected region containing the origin. 
(ii) Let $f(z)$ be analytic in $\mathcal{R}$. Then, for $\alpha$ and $\rho \in \mathbb{C}$ with $\operatorname{Re}(\rho)>-1$, the following relation holds true

$$
{ }_{0}^{\rho} D_{z}^{\alpha} f(z)=\frac{(\rho+1)^{\alpha+1}}{\Gamma(-\alpha)} z^{-(\rho+1)(\alpha+1)} \sum_{n=0}^{\infty} \frac{(\alpha+1) n}{n !} z^{-n(\rho+1)} D_{z}^{-1} z^{(\rho+1) n+\rho} f(z)
$$

Proof Applying Theorem 3 to Theorem 1, the result follows easily.

Corollary 5 Substituting $\rho=0$ in the Theorem 4, we obtain

$$
D_{z}^{\alpha} f(z)=\frac{z^{-(\alpha+1)}}{\Gamma(-\alpha)} \sum_{n=0}^{\infty} \frac{(\alpha+1)_{n}}{n !} z^{-n} D_{z}^{-1} z^{n} f(z)
$$

Example 2 Putting $f(z)=\mathrm{e}^{z}$ in Theorem 4 gives for the l.h.s. of (20)

$$
{ }_{0}^{\rho} D_{z}^{\alpha} \mathrm{e}^{z}=\sum_{k=0}^{\infty} \frac{{ }_{0}^{\rho} D_{z}^{\alpha} z^{k}}{k !}=(\rho+1)^{\alpha} z^{-\alpha(\rho+1)} \sum_{k=0}^{\infty} \frac{\Gamma\left(\frac{k}{\rho+1}+1\right)}{\Gamma\left(\frac{k}{\rho+1}+1-\alpha\right)} \frac{z^{k}}{k !} .
$$

Now, for the r.h.s. of (20), the computation of the fractional derivative operator applied to the exponential function yields

$$
D_{z}^{-1} z^{(\rho+1) n+\rho} \mathrm{e}^{z}=\sum_{k=0}^{\infty} \frac{D_{z}^{-1} z^{(\rho+1) n+\rho+k}}{k !}=z^{(\rho+1) n+\rho+1} \sum_{k=0}^{\infty} \frac{z^{k}}{(1+(\rho+1) n+\rho+k) k !}
$$

Replacing (22) and (23) in (20), we arrive, after some simplifications, to the next formula:

$$
\sum_{k=0}^{\infty} \frac{\Gamma\left(\frac{k}{\rho+1}+1\right) z^{k}}{\Gamma\left(\frac{k}{\rho+1}+1-\alpha\right) k !}=\frac{(\rho+1)}{\Gamma(-\alpha)} \sum_{n=0}^{\infty} \frac{(\alpha+1)_{n}}{n !} \sum_{k=0}^{\infty} \frac{z^{k}}{(1+(\rho+1) n+\rho+k) k !} .
$$

Note that by making use of Theorem 1 , we also have that

$$
\begin{aligned}
\sum_{k=0}^{\infty} \frac{\Gamma\left(\frac{k}{\rho+1}+1\right) z^{k}}{\Gamma\left(\frac{k}{\rho+1}+1-\alpha\right) k !}= & \frac{(\rho+1)}{\Gamma(-\alpha)} z^{-(\rho+1)} \sum_{n=0}^{\infty} \frac{(\alpha+1)_{n}}{n !} z^{-n(\rho+1)} \\
& \times \Gamma((\rho+1) n+\rho+1) \mathrm{e}^{z} D_{z}^{-(\rho+1) n-\rho-1} \mathrm{e}^{-z} \\
= & \frac{(\rho+1) \mathrm{e}^{z}}{\Gamma(-\alpha)} \sum_{n=0}^{\infty} \frac{(\alpha+1)_{n}}{(1+(\rho+1) n+\rho) n !} \sum_{k=0}^{\infty} \frac{(1)_{k}(-z)^{k}}{(2+(\rho+1) n+\rho)_{k} k !}
\end{aligned}
$$

\section{A Leibniz rule for the fractional derivative operator ${ }_{0}^{\rho} D_{z}^{\alpha}$}

This section is devoted to obtain a generalized Leibniz-type rule for the fractional derivative operator ${ }_{0}^{\rho} D_{z}^{\alpha}$. Two special cases are also computed. This is done by using the first representation (10) and the generalized Leibniz rule for fractional derivatives obtained by Osler [7] and given in the next theorem.

\section{Theorem 6}

(i) Let $\mathcal{R}$ be a simply connected region containing the origin. 
(ii) Let $f(z)$ and $g(z)$ be analytic in $\mathcal{R}$. Then, for $0<a \leq 1, \alpha \in \mathbb{C}$ with $\alpha$ not a negative integer, $\gamma \in \mathbb{C}$, the following Leibniz rule holds true

$$
D_{z}^{\alpha} f(z) g(z)=a \sum_{n=-\infty}^{\infty}\left(\begin{array}{c}
\alpha \\
\gamma+a n
\end{array}\right) D_{z}^{\alpha-\gamma-a n} f(z) D_{z}^{\gamma+a n} g(z)
$$

We are now able to establish the generalized Leibniz rule for the operator ${ }_{0}^{\rho} D_{z}^{\alpha}$.

\section{Theorem 7}

(i) Let $\mathcal{R}$ be a simply connected region containing the origin.

(ii) Let $f(z)$ and $g(z)$ be analytic in $\mathcal{R}$. Then, for $0<a \leq 1, \alpha \in \mathbb{C}$ with $\alpha$ not a negative integer, $\gamma \in \mathbb{C}, \operatorname{Re}(\rho)>-1$ and for all $k \in \mathbb{N}_{0},(-1-\rho-(\rho+1) k)$ not a negative integer, the following Leibniz rule

$$
\begin{aligned}
{ }_{0}^{\rho} D_{z}^{\alpha} f(z) g(z)= & a \frac{(\rho+1)^{\alpha+1}}{\Gamma(-\alpha)} z^{-(\rho+1)(\alpha+1)} \\
& \times \sum_{k=0}^{\infty} \frac{(\alpha+1)_{k} \Gamma(1+\rho+(\rho+1) k)}{k ! z^{(\rho+1) k}} \sum_{n=-\infty}^{\infty}\left(\begin{array}{c}
-1-\rho-(\rho+1) k \\
\gamma+a n
\end{array}\right) \\
& \times\left.\left. D_{z}^{-1-\rho-(\rho+1) k-\gamma-a n} f(w-z)\right|_{w=z} D_{z}^{\gamma+a n} g(w-z)\right|_{w=z}
\end{aligned}
$$

holds true.

Proof We know from Theorem 1 that the fractional derivative operator ${ }_{0}^{\rho} D_{z}^{\alpha}$ applied to the product of the functions $f(z) g(z)$ can be written in the following form:

$$
\begin{aligned}
{ }_{0}^{\rho} D_{z}^{\alpha} f(z) g(z)= & \frac{(\rho+1)^{\alpha+1}}{\Gamma(-\alpha)} z^{-(\rho+1)(\alpha+1)} \sum_{n=0}^{\infty} \frac{(\alpha+1) n}{n !} z^{-n(\rho+1)} \\
& \times\left.\Gamma((\rho+1) n+\rho+1) D_{z}^{-(\rho+1) n-\rho-1} f(w-z) g(w-z)\right|_{w=z},
\end{aligned}
$$

where $w \rightarrow z$ in the right side of (27) after the evaluation of the fractional derivative since the point $w$ must be near the point $z$. Applying Theorem 6 to the r.h.s. of (27) gives the desired result.

We end this paper by computing two special cases of Theorem 7 . Note that calculations have been done with the help of standard formulas for fractional derivative operator acting on common functions (see $[25,26])$.

Example 3 Setting $f(z)=1$ and $g(z)=\mathrm{e}^{z}$ in Theorem 7 gives after some simple calculations:

$$
\begin{aligned}
\sum_{j=0}^{\infty} \frac{\Gamma\left(\frac{j}{\rho+1}+1\right) z^{j}}{\Gamma\left(\frac{j}{\rho+1}+1-\alpha\right) j !}= & a \frac{(\rho+1) \mathrm{e}^{z}}{\Gamma(-\alpha)} \sum_{k=0}^{\infty} \frac{(\alpha+1)_{k} \Gamma(1+\rho+(\rho+1) k)}{k !} \\
& \times \sum_{n=-\infty}^{\infty} \frac{\left(\begin{array}{c}
-1-\rho-(\rho+1) k \\
\gamma+a n
\end{array}\right) F_{1}\left[\begin{array}{c}
1 ; \\
1-\gamma-a n ;
\end{array}\right]}{\Gamma(2+\rho+(\rho+1) k+\gamma+a n) \Gamma(1-\gamma-a n)}
\end{aligned}
$$


Example 4 If we put $f(z)=(1-z)^{-\mu}$ and $g(z)=(1-z)^{-v}$ in Theorem 7 , we obtain, after simplifications, the following relation:

$$
\begin{aligned}
\sum_{j=0}^{\infty} & \frac{(\mu+\nu)_{j} \Gamma\left(\frac{j}{\rho+1}+1\right) z^{j}}{\Gamma\left(\frac{j}{\rho+1}+1-\alpha\right) j !} \\
= & a \frac{(\rho+1)(1-z)^{-\mu-v}}{\Gamma(-\alpha)} \sum_{k=0}^{\infty} \frac{(\alpha+1)_{k} \Gamma(1+\rho+(\rho+1) k)}{k !} \sum_{n=-\infty}^{\infty}\left(\begin{array}{c}
-1-\rho-(\rho+1) k \\
\gamma+a n
\end{array}\right) \\
& \times \frac{{ }_{2} F_{1}\left[\begin{array}{c}
\mu, \\
2+\rho+(\rho+1) k+\gamma+a n ;
\end{array}\right]}{\Gamma(2+\rho+(\rho+1) k+\gamma+a n) \Gamma(1-\gamma-a n)} .
\end{aligned}
$$

\section{Competing interests}

The authors declare that they have no competing interests.

\section{Authors' contributions}

All authors completed the paper together. Both authors read and approved the final manuscript.

\section{Author details}

${ }^{1}$ Department of Mathematics and Computer Science, University of Quebec at Chicoutimi, Quebec, G7H 2B1, Canada.

${ }^{2}$ Department of Mathematics and Computer Science, Royal Military College, Kingston, Ontario K7K 5L0, Canada.

\section{Acknowledgements}

Dedicated to Professor Hari M Srivastava.

Authors wish to thank Professor HM Srivastava for valuable suggestions and comments.

\section{Received: 12 November 2012 Accepted: 26 March 2013 Published: 11 April 2013}

\section{References}

1. Podlubny, I: Fractional Differential Equations: An Introduction to Fractional Derivatives, Fractional Differential Equations, to Methods of Their Solution and Some of Their Applications. Academic Press, New York (1999)

2. Samko, SG, Kilbas, AA, Marichev, OI: Fractional Integrals and Derivatives: Theory and Applications. Gordon \& Breach, New York (1993)

3. Campos, LMBC: On a concept of derivative of complex order with application to special functions. IMA J. Appl. Math. 33, 109-133 (1984)

4. Campos, LMBC: On rules of derivation with complex order of analytic and branched functions. Port. Math. 43 347-376 (1985)

5. Campos, LMBC: On a systematic approach to some properties of special functions. IMA J. Appl. Math. 36, 191-206 (1986)

6. Nekrassov, PA: General differentiation. Mat. Sb. 14, 45-168 (1888)

7. Osler, TJ: Leibniz rule for the fractional derivatives and an application to infinite series. SIAM J. Appl. Math. 18,658-674 (1970)

8. Osler, TJ: Leibniz rule, the chain rule and Taylor's theorem for fractional derivatives. Ph.D. Thesis, New York University (1970)

9. Lavoie, J-L, Osler, TJ, Tremblay, R: Fundamental Properties of Fractional Derivatives via Pochhammer Integrals. Lecture Notes in Mathematics (1976)

10. Pochhammer, C: Uber ein integral mit doppeltem unlauf. Math. Ann. 35, 470-494 (1890)

11. Pochhammer, C: Uber eine classe von integral mit geschlossener integrationscurve. Math. Ann. 37, 500-511 (1890)

12. Pochhammer, C: Zur theorie der Eulers'chen integrale. Math. Ann. 35, 495-526 (1890)

13. Tremblay, R: Une contribution à la théorie de la dérivée fractionnaire. Ph.D. Thesis, Laval University, Canada (1974)

14. Tremblay, R, Fugère, BJ: The use of fractional derivatives to expand analytical functions in terms of quadratic functions with applications to special functions. Appl. Math. Comput. 187, 507-529 (2007)

15. Tremblay, R, Gaboury, S, Fugère, B-J: A new Leibniz rule and its integral analogue for fractional derivatives. Integral Transforms Spec. Funct. 24(2), 111-128 (2013)

16. Tremblay, R, Gaboury, S, Fugère, B-J: A new transformation formula for fractional derivatives with applications. Integral Transforms Spec. Funct. 24(3), 172-186 (2013)

17. Tremblay, R, Gaboury, S, Fugère, B-J: Taylor-like expansion in terms of a rational function obtained by means of fractional derivatives. Integral Transforms Spec. Funct. 24(1), 50-64 (2013)

18. Saxena, RK, Kiryakova, V, Dave, OP: A unified approach to certain fraction integration operators. Math. Balk. 8(2-3), 211-219 (1994)

19. Kilbas, AA, Srivastava, HM, Trujillo, JJ: Theory and Applications of Fractional Differential Equations. Elsevier Amsterdam (2006)

20. Katugampola, UN: New approach to fractional integral. Appl. Math. Comput. 218, 860-865 (2011) 
21. Kilbas, AA: Hadamard-type fractional calculus. J. Korean Math. Soc. 38(6), 1191-1204 (2001)

22. Butzer, PL, Kilbas, AA, Trujillo, JJ: Compostions of Hadamard-type fractional integration operators and the semigroup property. J. Math. Anal. Appl. 269, 387-400 (2002)

23. Butzer, PL, Kilbas, AA, Trujillo, JJ: Fractional calculus in the Mellin setting and Hadamard-type fractional integrals. J. Math. Anal. Appl. 269, 1-27 (2002)

24. Rainville, ED: Special Functions. Macmillan Co., New York (1960)

25. Erdelyi, A, Magnus, W, Oberhettinger, F, Tricomi, F: Tables of Integral Transforms, vol. I. McGraw-Hill, New York (1954)

26. Erdelyi, A, Magnus, W, Oberhettinger, F, Tricomi, F: Tables of Integral Transforms, vol. II. McGraw-Hill, New York (1954)

doi:10.1186/1029-242X-2013-167

Cite this article as: Gaboury et al.: Some relations involving a generalized fractional derivative operator. Journal of Inequalities and Applications 2013 2013:167.

Submit your manuscript to a SpringerOpen ${ }^{\circ}$ journal and benefit from:

- Convenient online submission

- Rigorous peer review

- Immediate publication on acceptance

- Open access: articles freely available online

- High visibility within the field

- Retaining the copyright to your article 